\title{
O RPG (roleplaying game) como ferramenta de ensino do suporte básico da vida
}

\author{
The RPG (roleplaying game) as a teaching tool in basic life support
}

\author{
César Henrique Morais Alves ${ }^{1}$, Aldemar Araujo Castro ${ }^{2}$
}

Alves CHM, Castro AA. O RPG (roleplaying game) como ferramenta de ensino do suporte básico da vida / The RPG (role-playing game) as a teaching tool in basic life support / The RPG (roleplaying game) as a teaching tool in basic life support. Rev Med (São Paulo). 2018 jan.-fev.;97(1):30-5.

RESUMO: Objetivo. Determinar a diferença de proporção de desempenhos satisfatórios nas avaliações de habilidades em Suporte Básico da Vida (SBV) em estudantes de medicina depois de utilizar o RPG (role-playing game) como ferramenta de ensino comparado com antes do RPG. A hipótese é que a diferença de proporção é de $10 \%$ a mais após o uso do RPG. Métodos. Tipo de estudo: antes-depois. Amostra: estudantes do curso de graduação em medicina, do primeiro ao último ano, estratificados por ano em uma amostra por conveniência. Procedimentos: Foram realizadas sessões de RPG simulando diferentes situações da parada cardiorrespiratória, com avaliação dos participantes imediatamente antes e depois destas sessões. Variável: a diferença de proporção de desempenhos satisfatórios nas avaliações de habilidades em SBV depois de utilizar o RPG como ferramenta de ensino comparado com antes. Foi utilizado um formulário padronizado para avaliar as habilidades e os conhecimentos. Método estatístico: o tamanho da amostra foi estimado em 77 participantes. A análise estatística foi realizada com o teste de McNemar, e foi calculado o intervalo de confiança de $95 \%$. Resultados. A diferença de proporção de desempenhos satisfatórios nas avaliações de habilidades em SBV depois de utilizar o RPG como ferramenta de ensino comparado com antes foi de 58\% (IC95\% 45 a 71, P < 0,0001). A diferença de proporção de desempenhos satisfatórios nas avaliações de conhecimentos sobre SBV depois de utilizar o RPG comparado com antes foi de $36 \%$ (IC95\% 24 a 48, P < 0,0001). Conclusões. O uso do RPG no ensino do SBV pode melhorar a aquisição de habilidades e conhecimentos em SBV.

Descritores: Jogos experimentais; Reanimação cardiopulmonar/ educação; Aprendizagem baseada em problemas; Estudantes de medicina.

\begin{abstract}
Background. Determining the satisfactory performances ratio difference in the abilities evaluations in Basic Life Support (BLS) in medicine students after using the RPG (role-playing game) as a teaching tool compared to before the RPG. The hypothesis is that the ratio difference is $10 \%$ higher after using the RPG. Methods. Design: before and after study. Sample: undergraduate students of the medicine course, from the first to the last year, stratified by year in a convenience sample. Procedures: RPG sessions were held to simulate different cardiorespiratory arrest situations, with the evaluation of the participants immediately before and after these sessions. Variable: satisfactory performances ratio difference in the abilities evaluations in Basic Life Support after using the RPG compared to before. A standardized formulary was used to evaluate the abilities and the knowledge. Statistical method: the size of the sample was estimated in 77 individuals. The statistical analysis was done with the McNemar's test; it was calculated a confidence interval of $95 \%$ for each estimated point. Results. The satisfactory performances ratio difference in the abilities evaluations in Basic Life Support in medicine students after using the RPG as a teaching tool compared to before was equal to $58 \%$ (CI95\% 45 a 71, $\mathrm{P}<0,0001)$. The satisfactory performances ratio difference in the knowledge evaluations in Basic Life Support in medicine students after using the RPG as a teaching tool compared to before was equal to $36 \%$ (CI95\% 24 a 48, P $<0,0001)$. Conclusions. Using the RPG in the BLS teaching can improve the acquisition of abilities and knowledge in BLS.
\end{abstract}

Keywords: Games, experimental; Cardiopulmonary resuscitation/ education; Problem-based learning; Students, medical.

1. Aluno do curso de graduação em Medicina do $11^{\circ}$ período da Universidade Estadual de Ciências da Saúde de Alagoas, Maceió, AL. Email: henrique.morais72@gmail.com; Orcid: http://orcid.org/0000-0001-8191-8240.

2. Professor Assistente, Mestre, da Universidade Estadual de Ciências da Saúde de Alagoas, Maceió, AL, BR. Orcid: http:// orcid.org/0000-0003-0212-5950.

Endereço para correspondência: Aldemar Araujo Castro. Rua Doutor Jorge de Lima 113. Trapiche da Barra. CEP: $57010-382$.

Maceió, AL, BR. Email: aldemararaujocastro@gmail.com 


\section{INTRODUÇÃO}

$\mathrm{O}$ RPG (do inglês, role-playing game) é um jogo de interpretação no qual os participantes vivenciam personagens que trabalham em equipe e vivem uma aventura imaginária que é narrada pelo mestre, o árbitro do jogo, responsável por descrever tudo o que acontece nos cenários, como o que os personagens veem, sentem ou ouvem. Frente à situação descrita, os jogadores devem decidir e descrever as ações a serem tomadas por seus respectivos personagens, cabendo ao mestre verificar o sucesso ou falha dessas ações através do teste das habilidades e da sorte de cada personagem, sendo essa última representada pela rolagem de dados ${ }^{1}$.

Algumas características do RPG criam a possibilidade de utilizá-lo como ferramenta de ensino. $\mathrm{O}$ RPG pode ser utilizado na formação de profissionais de saúde, que além de assimilar conceitos básicos, precisam ter o desenvolvimento da cooperatividade em sua formação, pois esses indivíduos normalmente trabalham em equipe. Além disso, a aleatoriedade introduzida pelos dados cria suspense e promove ludicidade, tornando o aprendizado prazeroso. A permanência das aulas expositivas como peça central do ensino na maioria das Universidades foi questionada por dois experimentos que chegaram a resultados que sugerem que métodos ativos de ensino aprendizagem, além se serem mais engajantes para o estudante, podem até certo ponto permitir retenção conhecimento de maneira mais eficiente que os métodos tradicionais $^{2}$. Posteriormente, um trabalho a respeito da aprendizagem por meio de role-playing games com estudantes de medicina concluiu que aulas com RPG podem ser tão eficientes quanto aulas tradicionais (expositivas), sendo bem aceitas pelos estudantes e permitindo uma retenção mais duradoura do conhecimento ${ }^{3}$. O uso do RPG para o ensino do SBV pode ser uma ferramenta eficaz para desenvolver as habilidades para situações do dia-a-dia.

Assim, é relevante responder a pergunta de pesquisa: qual a diferença de proporção de desempenhos satisfatórios nas avaliações de habilidades em Suporte Básico da Vida em estudantes de medicina depois de utilizar o RPG (roleplaying game) como ferramenta de ensino comparado com antes do RPG?

\section{MATERIAL E MÉTODOS}

O projeto de pesquisa foi aprovado pelo Comitê de Ética em Pesquisa (CAAE: 34724414.1.0000.5011). Foi realizado um estudo antes-depois. O local foi o Auditório Prof. Jurandir Boia Rocha, localizado na Universidade Estadual de Ciências da Saúde de Alagoas.

O tamanho da amostra foi calculado em 77 indivíduos. Foram incluídos os estudantes do curso de graduação em Medicina da Universidade Estadual de Ciências da Saúde de Alagoas, do primeiro ao último ano, estratificados por ano. Foram excluídos aqueles que se encontravam não ativos, aqueles que se declararam impossibilitados de participar da pesquisa e aqueles que se declararam portadores de doença cardiovascular, osteoarticular ou óssea. A amostra foi não probabilística por conveniência. O recrutamento ocorreu de 28 de março a 12 de dezembro de 2015 e foi realizado por convites em grupos específicos da universidade em mídias sociais. Foram preparadas duas cópias do TCLE para leitura, explicação e assinatura pelos candidatos e pelos pesquisadores.

$\mathrm{Na}$ intervenção foram realizadas as sessões (máximo de seis participantes) de RPG com sete etapas: 1) introdução (5 minutos); 2) avaliação de conhecimentos antes do uso do RPG (30 minutos) - prova objetiva com 23 questões de múltipla escolha; 3 ) avaliação de habilidades antes do uso do RPG (30 minutos) - avaliação das ações tomadas pelos participantes em uma simulação de parada cardiorrespiratória com manequins de RCP de acordo com a observação de 40 itens de um formulário padronizado (cada um sendo marcado como "não acertou", "acertou 50\%" e "acertou 100\%"); 4) aula com RPG (110 minutos) (http:// bit.ly/sbvrpg2017); 5) avaliação de conhecimentos depois do uso do RPG (30 minutos) - semelhante à primeira, mas com questões e alternativas em posições diferentes; 6) avaliação de habilidades depois do uso do RPG (30 minutos) - semelhante à primeira; e 7) encerramento (5 minutos).

As aulas de SBV com RPG eram baseadas na aventura de um personagem que era convidado por Hipócrates a aprender o SBV, viajando no tempo e espaço para diferentes situações. As avaliações de conhecimentos e habilidades foram baseadas na versão de 2010 das diretrizes ${ }^{4}$ da American Heart Association.

Os participantes interpretavam o personagem alternadamente e realizavam o SBV em manequins infláveis (Mini Anne ${ }^{\circledR}$ Plus, Laerdal, Wappingers Falls, $\mathrm{NY}$ ) com uso do desfibrilador externo automático de treinamento (AED Trainer with Multi-Language USB Port \& Metronome, American Red Cross ${ }^{\circledR}$, Washington, DC).

A variável primária foi a diferença de proporção de desempenhos satisfatórios nas avaliações de habilidades em Suporte Básico da Vida (SBV) depois do uso de RPG comparado com antes (H2 - H1).

As variáveis secundárias foram:

a) proporção de desempenhos satisfatórios na avaliação de habilidades em SBV antes do uso de RPG como ferramenta de ensino (H1);

b) proporção de desempenhos satisfatórios na avaliação de habilidades em SBV depois do uso de RPG como ferramenta de ensino (H2);

c) A diferença de proporção de desempenhos satisfatórios nas avaliações de conhecimentos sobre SBV depois do uso de RPG comparado com antes (P2 - P1);

d) proporção de desempenhos satisfatórios na avaliação de conhecimentos sobre SBV antes do uso de RPG como ferramenta de ensino (P1);

e) proporção de desempenhos satisfatórios na avaliação de conhecimentos sobre SBV depois do uso de RPG como ferramenta de ensino (P2). 
Foi considerado como satisfatório todo o desempenho que alcançou $70 \%$ ou mais de acerto nas questões da avaliação de conhecimentos ou habilidades. Os dados complementares foram: idade, sexo, praticante ou não de RPG e período do curso de graduação.

Método estatístico: o tamanho da amostra foi calculado em 77 indivíduos. Foi considerada a hipótese de que do total de participantes que tivessem desempenho insatisfatório antes do RPG, era esperado que $10 \%$ teriam desempenho satisfatório depois do RPG, enquanto que do total de participantes que tivessem desempenho satisfatório antes do RPG, era esperado que $0 \%$ teriam desempenho insatisfatório depois do RPG. Foram considerados também o nível de significância $(\alpha)$ igual a $5 \%$ e o poder do teste (1- $\beta$ ) igual a $80 \%$. Foi utilizada uma calculadora eletrônica disponível na Internet (http://www.statstodo. com/SSizMcNemar_Pgm.php), na qual os dados foram inseridos. Os dados coletados foram armazenados no Microsoft Excel, por entrada única, após análise dos dados pelo pesquisador principal e associado, sendo as divergências resolvidas por reunião de consenso. A análise estatística foi realizada com o teste de $\mathrm{McNemar}^{5}$, sendo calculado o intervalo de confiança de 95\% (IC95\%) para cada ponto estimado.

\section{RESULTADOS}

O período de recrutamento foi de 28 de março a 12 de dezembro de 2015. Neste período 78 participantes foram avaliados. Destes, 27 das provas de habilidades antes do uso de RPG e 24 das provas de habilidades depois do uso de RPG foram destruídas acidentalmente por danos na unidade de armazenamento que as continha, enquanto uma prova de habilidades depois do uso de RPG não pode ser avaliada porque um participante apresentou dores na mão e foi orientado a encerrar sua participação na pesquisa. Assim, o número final na prova de habilidades antes do uso de RPG foi de 51 participantes e na prova de habilidades depois do uso de RPG foi de 53 participantes. Nas avaliações de conhecimento o número de participantes foi de 78 antes e depois do uso de RPG.

A amostra coletada demonstra uma predominância de participantes do sexo feminino na pesquisa (54\%; IC95\% de 43 a 64), assim como de não praticantes de RPG (89,74\%; IC95\% de 81 a 95). A média geral das idades foi de 23 anos (DP 5,49; IC 95\% 21 a 25), sendo a média feminina igual a 22 anos e a masculina igual a 24 anos. A idade máxima foi de 61 anos para o sexo masculino e 28 para o feminino. A idade mínima foi de 18 anos para ambos os sexos.

A diferença de proporção de desempenhos satisfatórios nas avaliações de habilidades em SBV depois do uso de RPG comparado com antes foi de 58\% (IC95\% 45 a 71, P < 0,0001). As demais variáveis estão descritas na Tabela 2.

Tabela 1 - Características da amostra

\begin{tabular}{|c|c|c|c|c|}
\hline & & Quantidade & $\%$ & IC $95 \%$ \\
\hline \multirow{2}{*}{ Sexo } & Masculino & 36 & $46 \%$ & 35 a 57 \\
\hline & Feminino & 42 & $54 \%$ & 43 a 64 \\
\hline \multirow{12}{*}{ Período do Curso de Graduação } & $1^{\mathrm{o}}$ & 13 & $17 \%$ & 10 a 27 \\
\hline & $2^{\circ}$ & 6 & $8 \%$ & 3 a 16 \\
\hline & $3^{\circ}$ & 12 & $15 \%$ & 9 a 25 \\
\hline & $4^{\circ}$ & 1 & $1 \%$ & 0 a 8 \\
\hline & $5^{\circ}$ & 6 & $8 \%$ & 3 a 16 \\
\hline & $6^{\circ}$ & 7 & $9 \%$ & 4 a 18 \\
\hline & $7^{\circ}$ & 11 & $14 \%$ & 8 a 24 \\
\hline & $8^{\circ}$ & 7 & $9 \%$ & 4 a 18 \\
\hline & $9^{\circ}$ & - & - & - \\
\hline & $10^{\circ}$ & 11 & $14 \%$ & 8 a 24 \\
\hline & $11^{\circ}$ & - & - & - \\
\hline & $12^{\circ}$ & 4 & $5 \%$ & 2 a 13 \\
\hline \multirow{2}{*}{ Prática de RPG } & Praticante & 8 & $10 \%$ & 5 a 19 \\
\hline & Não praticante & 70 & $90 \%$ & 81 a 95 \\
\hline \multirow{2}{*}{$\begin{array}{l}\text { Participação até o fim da sessão } \\
\text { de RPG }\end{array}$} & Sim & 77 & $99 \%$ & 92 a 100 \\
\hline & Não & 1 & $1 \%$ & 0 a 7 \\
\hline
\end{tabular}

IC95\%: intervalo de confiança de 95\%; \%: proporção. 
Alves CHM, Castro AA. O RPG (roleplaying game) como ferramenta de ensino do suporte básico da vida.

Tabela 2 - Variáveis primária e secundária

\begin{tabular}{l|c|c|c|c|c}
\hline & $\begin{array}{l}\text { Quantidade de } \\
\text { desempenhos satisfatórios }\end{array}$ & $\mathbf{n}$ & $\mathbf{\%}$ & IC95\% & Valor de p \\
\hline H2 - H1 & - & - & 58 & 45 a 71 & $<0,0001$ \\
\hline H1 & 0 & 51 & 0 & 0 a 7 & $<0,0001$ \\
\hline H2 & 31 & 53 & 58 & 44 a 72 & $<0,0001$ \\
\hline P2 $-\mathbf{P 1}$ & - & - & 36 & 24 a 48 & $<0,0001$ \\
\hline P1 & 47 & 78 & 60 & 48 a 71 & $<0,0001$ \\
\hline P2 & 75 & 78 & 96 & 89 a 99 & $<0,0001$ \\
\hline
\end{tabular}

H1: proporção de desempenhos satisfatórios na avaliação de habilidades em Suporte Básico da Vida (SBV) antes do uso de RPG como ferramenta de ensino; H2: proporção de desempenhos satisfatórios na avaliação de habilidades em SBV depois do uso de RPG como ferramenta de ensino; H2 - H1: diferença de proporção de desempenhos satisfatórios nas avaliações de habilidades em SBV depois do uso de RPG comparado com antes; P1: proporção de desempenhos satisfatórios na avaliação de conhecimentos sobre SBV antes do uso de RPG como ferramenta de ensino; P2: proporção de desempenhos satisfatórios na avaliação de conhecimentos sobre SBV depois do uso de RPG como ferramenta de ensino; P2 - P1: diferença de proporção de desempenhos satisfatórios nas avaliações de conhecimentos sobre SBV depois do uso de RPG comparado com antes; $\mathbf{n}$ : número de participantes avaliados; IC95\%: intervalo de confiança de $95 \%$.

\section{DISCUSSÃO}

A hipótese de que a diferença de proporção de desempenhos satisfatórios nas avaliações de habilidades em SBV depois do uso de RPG comparado com antes seria de $10 \%$ foi rejeitada. O resultado encontrado de $58 \%$ (IC95\% 45 a 71, P<0,0001) de desempenhos satisfatórios depois do uso do RPG demonstrou que ocorreu ganho de habilidades com a aplicação da sessão. Desta forma, o uso do RPG como ferramenta de ensino pode trazer melhorias na aquisição de habilidades em suporte básico da vida. Os fatores como cansaço dos participantes e realização da segunda prova de habilidades pouco após a aula com RPG podem ter influenciado diretamente os resultados obtidos, não permitindo a extrapolação dos mesmos resultados para uma análise das habilidades em longo prazo. Por não ter sido realizado um ensaio randomizado não se pode ter certeza que essa diferença foi devido a sessão de RPG. No entanto, a pesquisa demonstrou que o RPG tem um potencial de ser uma ferramenta de ensino para o SBV e que merece ser testado por meio de um ensaio randomizado.

Foi possível verificar ganho de habilidades de $58 \%$ depois do uso do RPG. Outros estudos ${ }^{6-10}$ não avaliaram quantitativamente o ganho de habilidades dos participantes, restringindo-se à descrição do modo de uso do RPG em sala de aula, à avaliação da satisfação em participar de atividades com RPG e ao levantamento de hipóteses sobre as possíveis vantagens do uso deste método. Desta forma, medir o ganho de habilidades por meio do uso do RPG é fundamental para determinar a sua efetividade como ferramenta de ensino.

A proporção de desempenhos satisfatórios na avaliação de habilidades em Suporte Básico da Vida antes do uso de RPG foi de $0 \%$ (IC95\% 0 a $7, \mathrm{P}<0,0001$ ), evidenciando um baixo nível de habilidades prévio por parte da amostra selecionada. Foi possível observar uma discrepância entre as variáveis $\mathrm{P} 1 \mathrm{e}$ H1 (60\% versus $0 \%$, respectivamente). Este fato pode ser um reflexo de um sistema de ensino no qual os estudantes de medicina estão mais acostumados a aprimorarem seus conhecimentos do que suas habilidades em suporte básico de vida. $\mathrm{Na}$ universidade onde o estudo foi realizado, o ensino do SBV ocorre nos dois primeiros anos do curso, nas disciplinas de Habilidades Médicas I e II. A ausência de resultados satisfatórios nas provas de habilidades antes do uso de RPG em todos os anos do curso de graduação pode decorrer da carência de reciclagem das habilidades treinadas em sala de aula.

O ganho de conhecimento após o uso do RPG foi de $36 \%$. Isso demonstra que o uso do RPG como ferramenta de ensino para o suporte básico da vida pode trazer melhorias para a aquisição de conhecimentos. Outros estudos ${ }^{6,7,8,9,10}$ não avaliaram o ganho de conhecimento dos participantes de maneira formal, por meio de realização de testes antes e após o uso do RPG. A avaliação sistemática e estruturada do ganho de conhecimentos através do uso de RPG é indispensável para determinar a sua efetividade como ferramenta pedagógica. Esta ideia é semelhante à sugerida por $\mathrm{Amaral}^{6}{ }^{6}$ como o próximo passo na exploração deste tema.

É possível que o ensino do suporte básico da vida com RPG de maneira complementar às aulas convencionais nesta população alvo seja uma boa estratégia de ensino. Esta hipótese é corroborada no método de ensino para Biologia Celular ${ }^{3}$, que ao aplicar um questionário de opinião a 230 estudantes de medicina após utilizar o RPG constatou que $78 \%$ dos participantes consideravam as aulas baseadas em RPG uma ferramenta de ensino efetiva e que $55 \%$ dos participantes consideravam as aulas baseadas em RPG melhores que as aulas convencionais, mas que não as substituíam.

Criar uma estratégia eficaz para trazer as habilidades 
de SBV para situações da vida real é um desafio ${ }^{4}$ e deve se basear nas diretrizes mais atualizadas ${ }^{11}$. Por isso é relevante comparar o ensino do SBV com RPG comparado com o método tradicional para verificar o ganho de conhecimentos e de habilidades. Assim, sugerimos a realização de um ensaio randomizado para testar a hipótese de que a diferença de proporção de desempenhos satisfatórios nas avaliações de habilidades em Suporte Básico da Vida em estudantes de medicina utilizando o RPG (role-playing game) como ferramenta de ensino quando comparados com o método tradicional é de $20 \%$.

\section{Resumo da Pesquisa Proposta:}

Título. A diferença de proporção de desempenhos satisfatórios nas avaliações de habilidades em Suporte Básico da Vida em estudantes de medicina utilizando o RPG (role-playing game) como ferramenta de ensino: ensaio clínico randomizado.

Contexto. Os RPGs (role-playing games), ou jogos de interpretação, consistem numa atividade lúdica onde seus participantes se valem da imaginação para viver aventuras como personagens fictícios. O RPG pode ser usado como uma forma dinâmica de aprendizado que promove um aumento da capacidade de raciocínio e do trabalho em equipe. Testar como o RPG pode contribuir no aprendizado do suporte básico da vida é um desafio. Assim, é relevante responder a pergunta de pesquisa: qual a diferença de proporção de desempenhos satisfatórios nas avaliações de habilidades em Suporte Básico da Vida em estudantes de medicina utilizando o RPG (role-playing game) como ferramenta de ensino quando comparados com o método tradicional?

Objetivo. Determinar a diferença de proporção de desempenhos satisfatórios nas avaliações de habilidades em Suporte Básico da Vida em estudantes de medicina utilizando o RPG (role-playing game) como ferramenta de ensino quando comparados com o método tradicional. A hipótese é que a diferença de proporção é de $20 \%$.

Tipo de estudo. Ensaio clínico randomizado, aberto.

Amostra. Serão incluídos os estudantes do curso de graduação em medicina. Serão excluídos aqueles que se encontram não ativos, aqueles que se declararem impossibilitados de participar da pesquisa ou aqueles que sejam portadores de doença cardiovascular, osteoarticular ou óssea.

Procedimentos. Grupos estudados: Grupo RPG, indivíduos que aprendem o suporte básico de vida utilizando o RPG como ferramenta de ensino. Grupo Tradicional, indivíduos que aprendem utilizando o método tradicional de ensino.

Variáveis. Variável primária. Diferença de proporção de desempenhos satisfatórios nas avaliações de habilidades em Suporte Básico da Vida do grupo RPG comparado ao grupo tradicional. Variáveis secundárias. a) proporção de desempenhos satisfatórios na avaliação de habilidades em Suporte Básico da Vida no grupo RPG, b) proporção de desempenhos satisfatórios na avaliação de habilidades em Suporte Básico da Vida no grupo tradicional, c) diferença de proporção de desempenhos satisfatórios nas avaliações de conhecimentos sobre Suporte Básico da Vida do grupo RPG comparado ao grupo tradicional, d) proporção de desempenhos satisfatórios na avaliação de conhecimentos sobre Suporte Básico da Vida no grupo RPG, e) proporção de desempenhos satisfatórios na avaliação de conhecimentos sobre Suporte Básico da Vida no grupo tradicional. Dados complementares. Idade, sexo, praticante ou não de RPG, período do curso de graduação.

Método estatístico. O tamanho da amostra foi estimado em 124 (calculado em 62 para cada grupo), considerando a proporção no Grupo 1 igual a 70\%, a proporção no Grupo 2 igual a 90\%, o nível de significância igual a $5 \%$, o poder do teste de $80 \%$ e o teste de hipótese bicaudal. A análise estatística será realizada com o teste de qui-quadrado; será calculado o intervalo de confiança de $95 \%$ para cada ponto estimado.

\section{CONCLUSÕES}

O uso do RPG no ensino do SBV pode melhorar a aquisição de habilidades e conhecimentos em SBV.

Fontes de fomento: Universidade Estadual de Ciências da Saúde de Alagoas, Maceió, AL.

Aspectos Éticos: Não existem eventuais conflitos de interesse (profissionais, financeiros e benefícios diretos ou indiretos) que possam ter influenciado os resultados da pesquisa ou o conteúdo. Aprovado pelo parecer no $787.798 \mathrm{em}$ 21/08/2014 (CAAE: 34724414.1.0000.5011) pelo Comitê de Ética em Pesquisa da Universidade Estadual de Ciências da Saúde de Alagoas.

Participação no estudo: Ambos os autores participaram da concepção e delineamento do estudo; coleta, análise e interpretação de dados; redação e revisão do manuscrito; aprovação final da versão a ser publicada e revisão do manuscrito após submissão à revista.

\section{REFERÊNCIAS}

1. Saldanha AA, Batista JRM. A concepção do role-playing game (rpg) em jogadores sistemáticos. Psicol Ciênc Prof. 2009;29(4):700-17. Disponível em: http://www.scielo.br/ pdf/pcp/v29n4/v29n4a05.
2. McCarthy JP, Anderson L. Active learning techniques versus traditional teaching styles: two experiments from history and political science. Innov High Educ. 2000;24(4):279-94. doi: 10.1023/B:IHIE.0000047415.48495.05. 
3. Randi MAF, Carvalho HF. Aprendizagem através de roleplaying games: uma abordagem para a educação ativa. Rev Bras Educ Med. 2013;37(1):80-8. doi: 10.1590/S010055022013000100012 .

4. Berg RA, Hemphill R, Abella BS, Aufderheide TP, Cave DM, Hazinski MF, Lerner EB, Rea TD, Sayre MR, Swor RA. Part 5: adult basic life support: 2010 American Heart Association Guidelines for Cardiopulmonary Resuscitation and Emergency Cardiovascular Care. Circulation. 2010;122(18 Suppl 3):S685-S705. Review. Erratum in: Circulation. 2011 ;124(15):e402. doi: 10.1161/ CIRCULATIONAHA.110.970939.

5. McNemar Q. Note on the sampling error of the difference between correlated proportions or percentages. Psychometrika. 1947;12(2):153-7. doi: http://dx.doi. org/10.1007/BF02295996.

6. Amaral RR, Bastos HFBN. O roleplaying game na sala de aula: uma maneira de desenvolver atividades diferentes simultaneamente. Rev Bras Pesq Educ Cien. 2011;11(1):10322. Disponível em: https://seer.lcc.ufmg.br/index.php/rbpec/ article/view/2329.

7. Oliveira AA, Ribeiro SAB. Um modelo de role-playing game (rpg) para o ensino dos processos da digestão. Itinerarius Reflectionis. 2012;8(2). doi: https://doi.org/10.5216/rir. v2i13.22340.
8. Laureano MOL, Campos MLCB, Sassi FMC, Almeida MEF. Uso do role-playing game (rpg) como complemento didático no ensino de imunologia. Rev Ensino Bioquímica. 2017;15(1):64-81. doi: https://doi.org/10.16923/reb. v15i1.725.

9. Boas ACV, Macêna Jr. AG, Passos MM. Rpg pedagógico como ferramenta alternativa para o ensino de física no ensino médio. Cad Bras Ensino Física. 2017;34(2):372-403. doi: http://dx.doi.org/10.5007/2175-7941.2017v34n2p372.

10. Cook MP, Gremo M, Morgan R. We're just playing: the influence of a modified tabletop role-playing game on ela students' in-class reading. Simul Gaming. 2016;48(2):199218. doi: http://dx.doi.org/10.1177/1046878116684570.

11. Kleinman ME, Brennan EE, Goldberger ZD, Swor RA, Terry M, Bobrow BJ, Gazmuri RJ, Travers AH, Rea T. Part 5: Adult Basic Life Support and Cardiopulmonary Resuscitation Quality: 2015 American Heart Association Guidelines Update for Cardiopulmonary Resuscitation and Emergency Cardiovascular Care. Circulation. 2015;132(18 Suppl 2):S414-35. doi: 10.1161/CIR.0000000000000259.

12. Brasil. Conselho Nacional de Saúde. Resolução n ${ }^{\circ} 466$, de 12 de dezembro de 2012. Diretrizes e normas regulamentadoras de pesquisas envolvendo seres humanos. Diário Oficial da União, Brasília, 13 jun. 2013. Seção 1. Disponível em: http:// conselho.saude.gov.br/resolucoes/2012/Reso466.pdf. 